\title{
Clinical Efficacy of Carbon Ion Radiotherapy for Unresectable Chondrosarcomas
}

\author{
REIKO IMAI ${ }^{1}$, TADASHI KAMADA ${ }^{1}$, NOBUHITO ARAKI ${ }^{2}$ and \\ THE WORKING GROUP FOR BONE and SOFT-TISSUE SARCOMAS \\ ${ }^{1}$ Hospital of the National Institute of Radiological Sciences, \\ Quantum and Radiological Science and Technology, Chiba, Japan; \\ ${ }^{2}$ Department of Orthopedic Surgery, Osaka Medical Center for Cancer and Cardiovascular Diseases, Osaka, Japan
}

\begin{abstract}
Background: Carbon ion radiotherapy has precise dose distribution and high biological effectiveness and is likely a promising therapy for patients with sarcomas. We evaluated the outcomes of carbon ion radiotherapy in patients with unresectable chondrosarcomas. Patients and Methods: A retrospective analysis of 75 tumors in 73 patients treated with carbon ion radiotherapy was performed. There were 26 spinal, 38 pelvic, and 11 other types of tumors. Seventy conventional and five dedifferentiated chondrosarcomas were treated in 69 and four patients, respectively. Results: The median follow-up period was 49.4 ( range $=6.4-146.4$ ) months. The 5-year local control, overall survival, and disease-free survival rates were 53\%, 53\%, and 34\%, respectively. By multivariate analysis, tumor volume and histological grade were significantly related to overall and disease-free survival. Five patients required surgical intervention because of adverse events in the bones. Conclusion: Carbon ion radiotherapy might be a treatment option for unresectable chondrosarcoma.
\end{abstract}

The curative treatment for chondrosarcoma is surgical resection (1-4). Complete removal of the tumor with negative margins ensures a successful outcome (2-4). Chondrosarcomas in the extremities are amenable to curative treatment more readily than those involving axial bones, such as tumors in the spine and pelvis. In 45 patients with only locally advanced tumors that were judged to be unresectable, van Maldegem et al. found a 3-year overall survival (OS) rate of $26 \%$ (1). There are reports that chemotherapy improved the survival rate in patients with

Correspondence to: Reiko Imai, MD, Ph.D. Hospital of the National Institute of Radiological Sciences, Quantum and Radiological Science and Technology, 4-9-1, Anagawa, Inage, Chiba 263-8555, Japan. Tel: +81 432063306, e-mail: imai.reiko@qst.go.jp

Key Words: Carbon ion radiotherapy, charged particle therapy, chondrosarcoma, sarcoma, bone and soft-tissue sarcoma. dedifferentiated chondrosarcomas; however, conventional chondrosarcoma is generally considered resistant to chemotherapy (5-7). Chondrosarcoma is also a radioresistant tumor, but Andreou et al. reported that patients with metastatic chondrosarcoma who received treatment with radiotherapy (RT) survived longer than those who received best supportive care (6). Carbon ion radiotherapy (C-ion RT) for unresectable sarcomas was introduced in 1996 at our Institute (8). Between 1996 and March 2015, over than 1,000 patients with bone and soft-tissue sarcomas were treated with C-ion RT at a single institute (9). In this study, we analyzed the results of patients with chondrosarcoma treated with C-ion RT according to our protocol.

\section{Patients and Methods}

Carbon ion radiotherapy. Carbon ion beams were generated by a synchrotron, the Heavy Ion Medical Accelerator in Chiba, Japan (HIMAC). Accelerated energies of 290, 350, 400, and $430 \mathrm{MeV} / \mathrm{n}$ were available. For axial tumors, 350 and $400 \mathrm{MeV} / \mathrm{n}$ beams were generally used. These energy beams had a range of $15-28 \mathrm{~cm}$ waterequivalent depth. The Bragg peak of the beams was modulated to fit the tumor shape using a pair of wobbler magnets, beam scatters, ridge filters, multi-leaf collimators, and compensation boluses. A respiratory gating system was employed. Details of the C-ion RT technique with HIMAC have been described previously (8-10).

In order to immobilize patients, low-temperature thermoplastic shells and body rests were used. A set of 1-5-mm-thick computed tomographic (CT) images was acquired for treatment planning. Magnetic resonance imaging (MRI) and CT images using contrast medium, and, if available, methionine or fluorodeoxyglucose positron-emission tomographic images were used to determine target volumes. The clinical target volume (CTV) included the tumor's potential area of spread. The planning target volume (PTV) basically included the CTV with an additional 5-mm margin. Irradiation was performed with a minimum of three ports. One port per day was used, and treatment was performed on four consecutive days a week from Tuesday to Friday. According to the protocol, the total dose was 64.0, 70.4, or 73.6 Gy [relative biological effectiveness (RBE)] in 16 fractions. Some spinal tumors abutting the spinal cord received 64.0 Gy (RBE). After 73.6 Gy (RBE) in 16 fractions was employed 
in the early stage, it was cancelled due to the development of severe late events. As a workable dose with minimum adverse events, 70.4 Gy (RBE) in 16 fractions was selected. All patients signed an informed consent form, approved by the local Institutional Review Board as 9601, 9901 and 9901(2) protocols on carbon ion radiotherapy for bone and soft-tissue sarcoma.

Follow-up and statistical analysis. The follow-up period was counted from the day of the first C-ion RT treatment session. Patients were monitored via physical examinations, CT, and MRI. Initial follow-up imaging examinations were performed after completion of all C-ion RT sessions, and they were conducted every 3-4 months thereafter, generally using CT and MRI with contrast medium in an alternating fashion. In cases where patients were unable to travel to our hospital, their latest medical reports and CT/MRI images were sent to us by their local hospitals. Local control (LC) was defined as no increase in tumor volume observed in two consecutive MRI or CT scans. Toxicities attributable to RT were scored using the Common Toxicity Criteria, version 2.0 (United States National Cancer Institute, Bethesda, MD, USA) (11).

The overall survival (OS), disease-free survival (DFS), and LC rates were determined by Kaplan-Meier method using Prism 5 (version 5.0; GraphPad Software Inc., San Diego, CA, USA). The log-rank test was used for individual comparisons using the same software. Multivariate analysis was performed by IBM SPSS statistics version 23 (IBM Corp., Armonk, NY, USA). A value of $p<0.05$ was considered statistically significant.

\section{Results}

Patients. Between June 2000 and February 2012, 73 patients with 75 tumors of chondrosarcoma were enrolled in a single $\mathrm{C}$-ion RT treatment protocol for bone and soft-tissue sarcomas located below C2 at our Institution. Pathologies of all tumors were reviewed by the central pathologist at our Institute. The patients consisted of 42 males and 31 females, with a median age of 57 years (range $=17-77$ years). All patients were followed-up for at least 2 years or until death. Surgical resection was not planned for any of the tumors; investigating the combination of surgery and C-ion RT was not the purpose of this study. Details of patient and tumor characteristics are summarized in Table I. Patients with metastatic disease at the time of referral to our hospital were not enrolled under this protocol; however, it was decided that patients with solitary metastasis appearing after a considerable time had elapsed since their last treatment could be included. Three patients with such a metastasis had received C-ion RT. The median follow-up period was 49 months for the 73 patients who survived for 6-146 months. All patients were followed-up for at least 2 years or until death.

Tumor control. Figure 1 shows MRI images of the tumor response after $\mathrm{C}$-ion RT. Of 75 tumors, 32 showed local recurrence. The 5-year LC rate of all evaluated tumors was $53 \%$ (Figure 2), and the median LC period was 39.6 months. The median LC period was 66 months in those with grade
Table I. Patient characteristics (73 patients with 75 lesions).

\begin{tabular}{lc}
\hline Characteristic & Value \\
\hline Median age (range), years & $57(17-77)$ \\
Male:female, no. of patients & $42: 31$ \\
Median tumor size (range), $\mathrm{cm}^{3}$ & $471(25-2900)$ \\
Tumor type, no. of lesions & \\
Primary tumor with no prior surgery & 55 \\
Recurrent tumor after resection & 17 \\
Metastatic tumor & 3 \\
Irradiation site, no. of lesions & \\
Spine (cervix/thorax/lumber/sacrum) & $26(3 / 5 / 4 / 14)$ \\
Pelvis (iliac/pubic/ischium) & $38(36 / 1 / 1)$ \\
Paraspinal region & $1 *$ \\
Rib & 6 \\
Scapula & 2 \\
Sternum & 1 \\
Femur & 1 \\
Histology, no. of patients & \\
Conventional chondrosarcoma & 69 \\
Grade 1 & 14 \\
Grade 2 & 51 \\
Grade 3 & 4 \\
Dedifferentiated & 4 \\
Total irradiated dose [Gy(RBE) in & \\
16 fractions], no. of lesions & \\
64.0 & 4 \\
70.4 & 29 \\
73.6 & \\
\hline
\end{tabular}

RBE: Relative biological effectiveness. *Metastatic.

G1, 40 in those with G2, 25 in those with G3 and nine in dedifferentiated chondrosarcoma tumors. According to histology, the 5-year LC rates were 58\% in those with G1 and $55 \%$ in those with G2. However, using log-rank test among all combinations of the two different grade groups, there were no significant differences (Figure 3 ). The study group was divided into two groups according to tumor size, with a cutoff of $470 \mathrm{~cm}^{3}$ (median volume). There was a significant difference in LC rates between these two groups by univariate analysis (Figure 4). Seven tumors less than $100 \mathrm{~cm}^{3}$ showed no recurrence, and the median LC was 65 months. Of the 17 tumors less than $200 \mathrm{~cm}^{3}$, recurrence developed in two tumors, and the median LC was 101 months.

Survival. The median follow-up period was 49 months for the 73 patients who survived for 6-146 months. Forty patients died and 30 patients survived for over 5 years. The median survival of the patients who died was 28.7 months, ranging from 6.4 to 124 months. The 5-year OS rate was 53\% (Figure 2 ). The OS rates based on histology are shown in Figure 5. The median survival of patients with G1 chondrosarcomas was 86 months, 49 months for those with G2 chondrosarcomas, 28 months for those with G3 conventional 

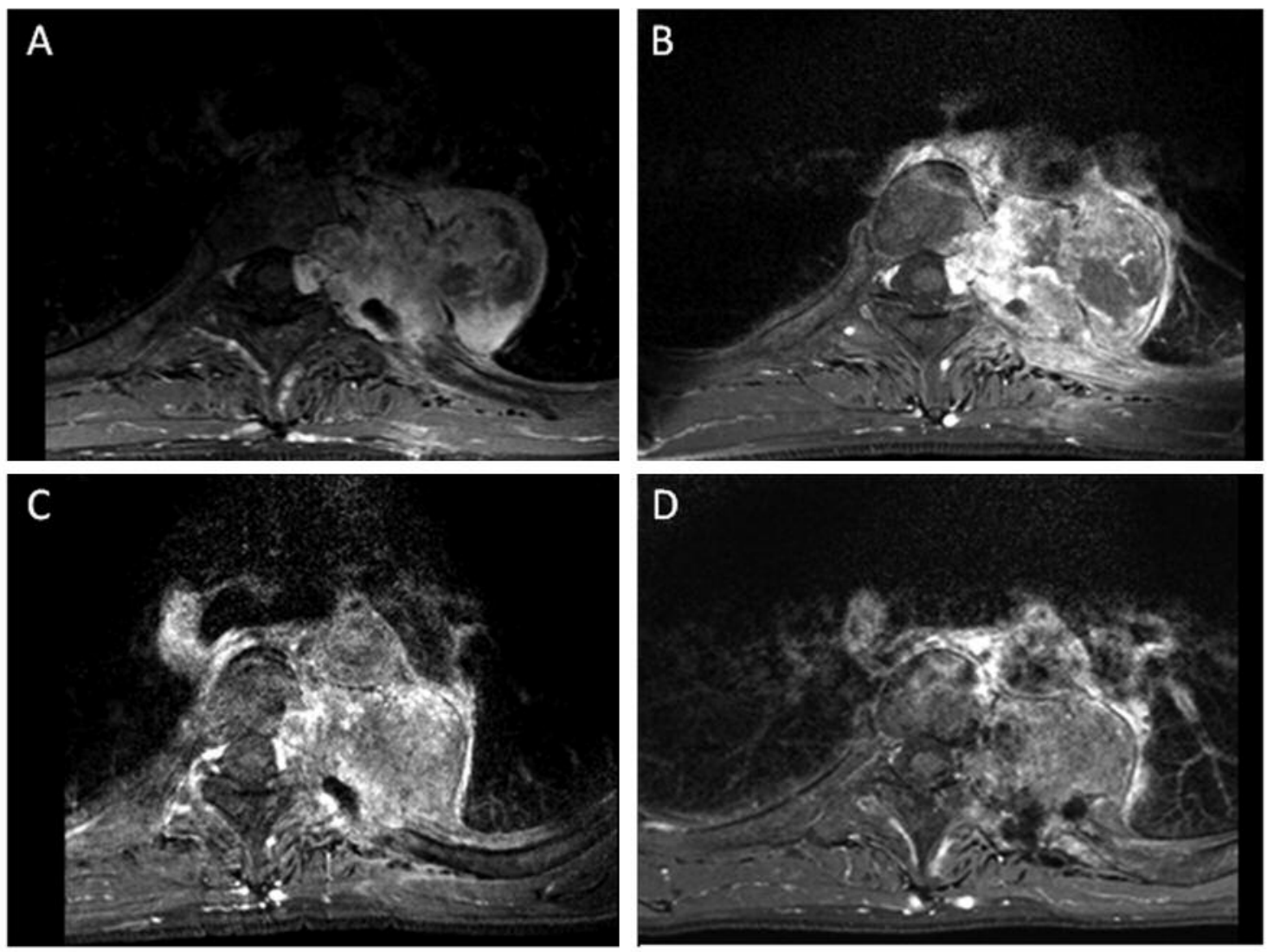

Figure 1. A 43-year-old man with chondrosarcoma of the left fourth rib. A: The tumor on T1-weighted magnetic resonance imaging with contrast enhancement before carbon ion radiotherapy. B: One year later, the size of the tumor was unchanged, but the enhancement by contrast medium had decreased. C: Three years later, the tumor was shrinking. D: Ten years later, the tumor had shrunk and the patient remained disease-free.

chondrosarcomas, and 10 months for those with dedifferentiated chondrosarcomas. Using the log-rank test among all combinations of two different grade groups, there were significant differences between the G1 group and the G3 and dedifferentiated chondrosarcoma group, and between the G2 and the latter group. There was no significant difference between those with G1 and G2 chondrosarcoma. There was no significant difference in OS rate between the patients with tumor status such as locally recurrent tumors after surgery and those with primary tumors; however, the difference between patients with large versus small tumors (cut-off of $470 \mathrm{~cm}^{3}$ ) was significant by univariate analysis (Figure 4). By multivariate analysis, tumor volume, and histological grade (G1 and G2, G3 and dedifferentiated) were significantly related to OS. However, tumor location (spine and other, pelvis), tumor status (primary, recurrence and metastasis) and age (cut-off of 65 years) were not related. The median DFS for all patients was 33 months. The 5-year DFS rate was $34 \%$ (Figure 2). At the time of evaluation, 22 patients were alive and free of disease. Multivariate analysis revealed that tumor volume and histology were significantly

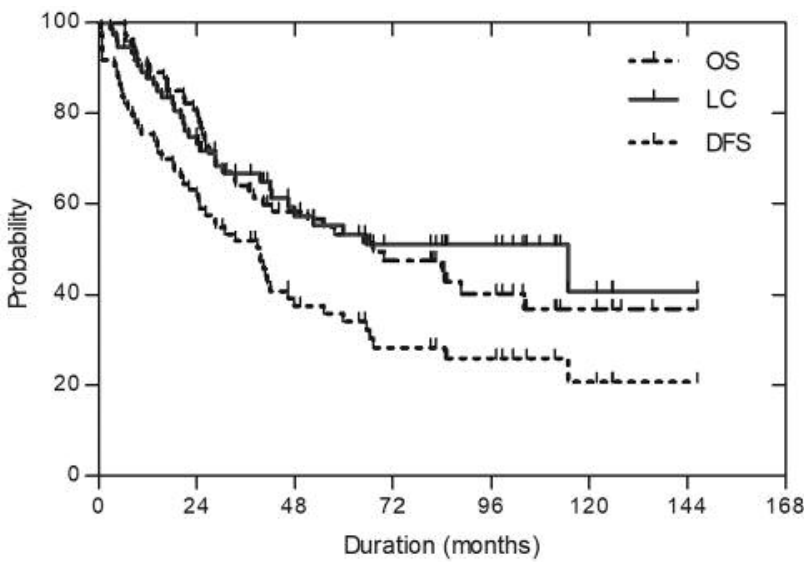

Figure 2. Local control (LC), overall survival (OS), and disease-free survival (DFS) rates for the whole group of 73 patients with 75 chondrosarcomas. The 5-year rates were 53\%, 53\%, and 34\%, respectively.

related to DFS. In 21 out of the 51 patients who either had disease or died, the site of first recurrence was that of the tumor originally irradiated with C-ion RT. 


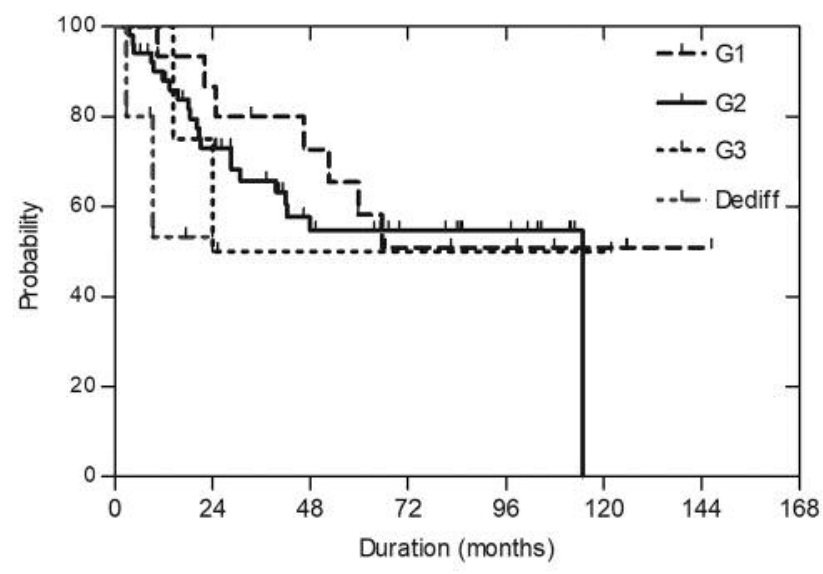

Figure 3. Local control rates according to histology. Of 15 lesions of G1 chondrosarcoma, the 5-year local control $(L C)$ rate was $58 \%$. Of 51 lesions of G2 chondrosarcoma, the 5-year LC rate was 55\%. Of four lesions of G3 chondrosarcoma, three had local recurrence before death, and of five lesions of de-differentiated chondrosarcoma two had local recurrence before death.

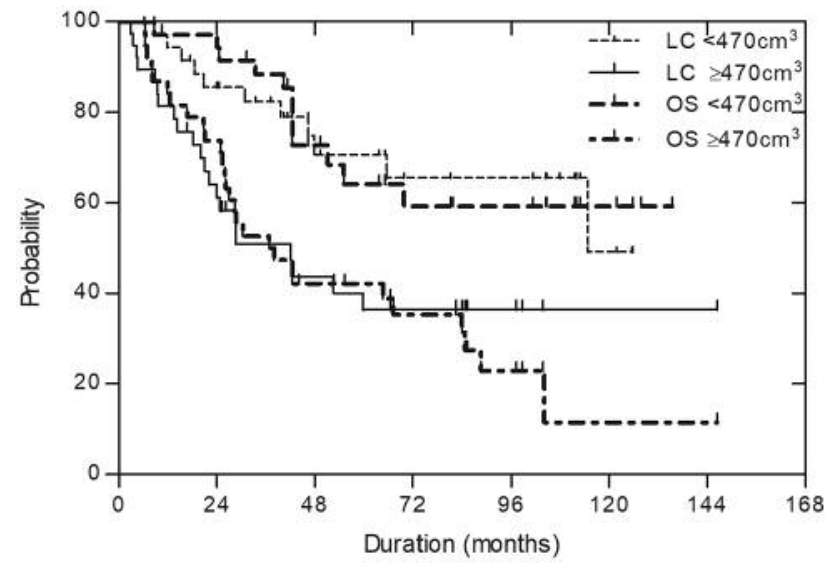

Figure 4. Local control (LC) and overall survival (OS) rates according to tumor size. The 73 patients and 75 lesions were divided into two groups according to tumor size: above or below the median tumor volume of $470 \mathrm{~cm}^{3}$. The large tumor group consisted of 38 lesions in 38 patients and the small tumor group of 37 lesions in 35 patients. There were significant differences in LC and OS rates (at $p=0.009$ and $p=0.0008$, respectively) between the two groups as determined by univariate analysis.

Adverse events. Late adverse events grade 3 and higher were observed in eight patients. Three patients had grade 3 late skin reactions; the remaining five had tumor invasion into the bone, where degeneration was inevitable after C-ion RT. One patient had a femoral pathological bone fracture requiring surgery. Two patients had femoral neck fractures, requiring

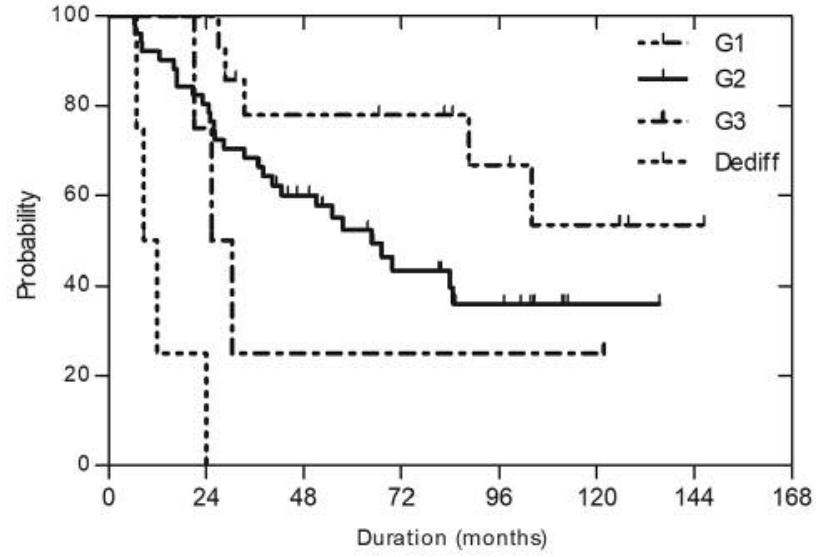

Figure 5. Overall survival rates according to histology. Of 14 patients with G1 chondrosarcoma, the 5-year survival rate was $77.9 \%$. Of 51 patients with G2 chondrosarcoma, the 5-year survival rate was $52.9 \%$. Of four patients with $G 3$ chondrosarcoma, three died within 30 months, and all four patients with dedifferentiated chondrosarcoma died within 24 months.

surgery, where no tumor invasion was evident, and one patient had femoral head necrosis also unrelated to tumor invasion, likewise requiring surgery. Another patient required a metal implant for a lumbar spinal compression fracture.

\section{Discussion}

One-third of chondrosarcomas occur in the pelvis, sacrum, and mobile spine $(12,13)$. Bergh et al. reported that the 5year OS rate of patients with 69 axial chondrosarcomas treated with surgery, including seven that were not resected, was $72 \%$ (3). For patients with pelvic chondrosarcomas, 5-year OS rates were reported to range from $60 \%$ to $80 \%$ in surgical groups $(2,4,14)$. In those reports, tumor grade was determined to be the most notable predictor of survival $(2-4,6)$. The survival rate in our study was worse compared to that of surgically treated patients. The most likely reason for this is that our group included patients with unresectable axial chondrosarcomas. Of our cases, $35 \%$ were spinal chondrosarcomas close to the spinal cord, or sacral lesions. Another suggested reason is that our patients were older than those in groups undergoing surgery. The median age of patients in the present study was 57 years, with $27 \%$ being over 65 years old, whereas patients in surgical studies were reported to be in their 40s $(2-4,6,14)$. van Maldegem et al. reported the outcome of unresectable conventional central chondrosarcoma treatment; in 45 patients with only locally advanced tumors judged to be unresectable, the 3-year OS 
rate was $26 \%$ (1). In their report, better OS was correlated with age $<40$ years and grade II tumor. In our series of 73 patients, the 3 -year OS rate of $63 \%$ was much better than that reported by van Maldegem et al. (1). Better OS was related to tumor grade, as it was in their study. Another factor that significantly predicted LC and OS for patients treated with $\mathrm{C}$-ion $\mathrm{RT}$ was tumor volume. By multivariate analysis, LC and OS rates were significantly related to tumor volume, using a cutoff of $470 \mathrm{~cm}^{3}$. In the group with small tumors, 5-year LC and OS were $71 \%$ and $64 \%$, respectively, results similar to those for resectable cases. This is consistent with previous studies of C-ion RT for osteosarcoma (15).

Only one case of femoral chondrosarcoma was treated with C-ion RT in our study group. The patient adamantly refused surgery, despite it having been recommended because the tumor was resectable and bone fracture after $\mathrm{C}$ ion RT was expected. After C-ion RT, the patient underwent surgery to prevent fracture. However, 25 months after surgery, a fracture occurred, requiring additional surgery. At the time of evaluation, the patient had been continuously disease-free for 85 months and used two crutches to walk. We do not consider sarcomas of the extremities, especially bone sarcomas, suitable for C-ion RT, as there is a high possibility of fracture in irradiated long bones, which would require surgery.

Dedifferentiated chondrosarcoma has a poor prognosis, with median survival reported to be 6-9 months $(5,16)$ Fifty percent of patients had local recurrence after surgery (5). In that report, five patients with pelvic tumors underwent surgery; two underwent amputation and died at 42 and 37 weeks from the date of diagnosis (5). In four patients with dedifferentiated chondrosarcoma in our study, the survival period was between 6.9 and 24.0 months from the first day of irradiation, with two patients having no local recurrence and remaining ambulatory, then dying of systemic metastases. Mitchell et al. reported that chemotherapy contributed to improvement of prognosis in their study; however, chemotherapy alone cannot be a curative treatment (5). Considering both the results of surgery and C-ion RT, the latter could be an option if the tumor is on the boundary of concern regarding complete or incomplete resection. G3 conventional chondrosarcoma has a prognosis similar to that of dedifferentiated chondrosarcoma, and therefore $\mathrm{C}$-ion RT can be included as a treatment option for these high-grade chondrosarcomas (17). C-ion RT should henceforth be considered a viable treatment option for patients with chondrosarcomas that orthopedic surgeons deem unresectable.

\section{Conflicts of Interest}

None.

\section{Acknowledgements}

This study was supported by the Research Project with Heavy Ions at NIRS-HIMAC of the National Institute of Radiological Sciences. The Authors extend their appreciation to all members of the Working Group for Bone and Soft-Tissue Sarcomas for their cooperation. Group members were Noriaki Kameda, Satoshi Abe, Yukihide Iwamoto, Toshifumi Ozaki, Chihiro Kanehira, Mitsunori Kaya, Kazuhisa Takahashi, Hirokazu Chuman, Hirohiko Tsujii, Masazumi Tsuneyoshi, Yoshihiro Nishida, Hiroaki Hiraga, Toru Hiruma, Rikuo Machinami, Akihiko Matsumine, Seiichi Matsumoto, Hideo Morioka, Takehiko Yamaguchi, and Tsukasa Yonemoto.

\section{References}

1 van Maldegem AM, Gelderblom H, Palmerini E, Dijkstra SD, Gambarotti M, Ruggieri P, Nout RA, van de Sande MA, Ferrari C, Ferrari S, Bovée JV and Picci P: Outcome of advanced, unresectable conventional central chondrosarcoma. Cancer 120: 3159-3164, 2014.

2 Pring ME, Weber KL, Unni KK and Sim FH: Chondrosarcoma of the pelvis. A review of sixty-four cases. J Bone Joint Surg Am 83-A: 1630-1642, 2001.

3 Bergh P, Gunterberg B, Meis-Kindblom JM and Kindblom LG: Prognostic factors and outcome of pelvic, sacral, and spinal chondrosarcomas: a center-based study of 69 cases. Cancer 91 : 1201-1212, 2001.

4 Deloin X, Dumaine V, Biau D, Karoubi M, Babinet A, Tomeno $B$ and Anract P: Pelvic chondrosarcomas: surgical treatment options. Orthop Traumatol Surg Res 95: 393-401, 2009.

5 Mitchell A, Ayoub K, Mangham D, Grimer R, Carter S and Tillman R: Experience in the treatment of dedifferentiated chondrosarcoma. J Bone Joint Surg 82: 55-61, 2000.

6 Andreou D, Ruppin S, Fehlberg S, Pink D, Werner M and Tunn PU: Survival and prognostic factors in chondrosarcoma: results in 115 patients with long-term follow-up. Acta Orthop 82: 749$755,2011$.

7 Gelderblom H, Hogendoorn PC, Dijkstra SD, van Rijswijk CS, Krol AD, Taminiau AH and Bovée JV: The clinical approach towards chondrosarcoma. Oncologist 13: 320-329, 2008.

8 Kamada T, Tsujii H, Tsuji H, Yanagi T, Mizoe JE, Miyamoto T, Kato H, Yamada S, Morita S, Yoshikawa K, Kandatsu S and Tateishi A: Efficacy and safety of carbon ion radiotherapy in bone and soft tissue sarcomas. J Clin Oncol 20: 4466-4471, 2002.

9 Kamada T, Tsujii H, Blakely EA, Debus J, De Neve W, Durante M, Jäkel O, Mayer R, Orecchia R, Pötter R, Vatnitsky S and Chu WT: Carbon ion radiotherapy in Japan: an assessment of 20 years of clinical experience. Lancet Oncol 16: e93-e100, 2015.

10 Imai R, Kamada T, Tsujii H, Sugawara S, Serizawa I and Tatezaki S: Effect of carbon ion radiotherapy for sacral chordoma: results of phase I-II and phase II clinical trials. Int J Radiat Oncol Biol Phys 77: 1470-1476, 2010.

11 National Cancer Institute. Common Toxicity Criteria, Version 2.0. National Cancer Institute: Bethesda, MD, 1999.

12 Campanacci M: Chondrosarcomas: In: Bone and Soft-Tissue Tumors. Springer Verlag: Vienna, pp. 265-343, 1990. 
13 Pritchard DJ, Lunke RJ, Taylor WF, Dahlin DC and Medley BE: Chondrosarcoma: a clinicopathologic and statistical analysis. Cancer 45: 149-157, 1980.

14 Guo W, Li D, Tang X and Ji T: Surgical treatment of pelvic chondrosarcoma involving periacetabulum. J Surg Oncol 101: 160-165, 2010.

15 Matsunobu A, Imai R and Kamada T: Imaizumi T, Tsuji H, Tsujii $\mathrm{H}$, Shioyama Y, Honda $\mathrm{H}$ and Tatezaki S: Impact of carbon ion radiotherapy for unresectable osteosarcoma of the trunk. Cancer 118: 4555-4563, 2012.
16 Mercuri M, Picci P, Campanacci L and Rulli E: De-differentiated chondrosarcoma. Skeletal Radiol 24: 409-416, 1995.

17 Wirbel RJ, Schulte M, Maier sB, Koschnik M and Mutschler WE: Chondrosarcoma of the pelvis: oncologic and functional outcome. Sarcoma 4: 161-168, 2000.

Received September 9, 2017

Revised October 6, 2017

Accepted October 12, 2017 